\title{
Cuidado integrado do paciente periodontal diabético tipo 2 na Atençẫo Básica: revisão scoping
}

\author{
I ${ }^{1}$ Lívia Maria Lopes de Oliveira, ${ }^{2}$ Lívia Mirelle Barbosa, ${ }^{3}$ Jhony Herick Cavalcanti \\ Nunes Negreiros, ${ }^{4}$ Luiz Alexandre Moura Penteado, ${ }^{5}$ Viviane Vanessa Rodrigues \\ da Silva Santana Lima I
}

Resumo: Objetivo: Investigar a existência de protocolos validados para o cuidado do paciente periodontal com diabetes mellitus tipo 2 (DM2), a fim de transformá-los em recomendaçóes para açôes que poderiam ser desenvolvidas no âmbito da atenção primária em saúde. Métodos: Foi realizada uma revisão scoping, para a qual foi revista a literatura publicada de 2012 a 2019 nas bases de dados PubMed, Scielo, LILACS, Web of Science e Scopus, nas línguas inglesa, espanhola e portuguesa. Resultados: Foram encontrados 236 títulos, dos quais 37 foram selecionados para leitura integral. Os artigos foram agrupados em quatro eixos, de acordo com a similaridade de abordagens para o assunto tratado. Conclusão: Recomenda-se desenvolver estudos que investiguem o impacto da integração da assistência médica e odontológica no controle glicêmico de pacientes com DM2 e validem os protocolos clínicos para a assistência multidisciplinar desse paciente no contexto da atenção primária em saúde.

> Palavras-chave: integralidade em saúde; diabetes mellitus tipo 2; doenças periodontais; protocolos clínicos; Atenção Primária em Saúde.

\author{
${ }^{1}$ Programa de Pós-Graduação \\ em Odontologia, Universidade \\ Federal de Pernambuco. Recife- \\ PE, Brasil (livialopesperiodontia@ \\ gmail.com). \\ ORCID: 0000-0002-7579-9254 \\ 2 Universidade de Pernambuco, \\ Programa de Pós-Graduação em \\ Odontologia. Recife-PE, Brasil \\ (dra.liviabarbosa@gmail.com). \\ ORCID: 0000-0002-8992-2890 \\ ${ }^{3}$ Programa de Pós-Graduação em \\ Odontologia, Universidade Federal \\ de Pernambuco. Recife-PE, Brasil \\ (jhonyherick@gmail.com). \\ ORCID: 0000-0002-3407-1021 \\ ${ }^{4}$ Universidade Federal de \\ Alagoas, Escola de Enfermagem. \\ Maceió-AL, Brasil (jhonyherick@ \\ gmail.com). \\ ORCID: 0000-0001-6683-7450 \\ ${ }^{5}$ Universidade Federal de Alagoas, \\ Escola de Enfermagem. Maceió- \\ AL, Brasil (professoravivisantana@ \\ gmail.com). \\ ORCID: 0000-0001-6903-1444
}




\section{Introdução}

A doença periodontal (DP) é uma condição crônica, microbiologicamente iniciada, na qual um processo de desregulaçấo imuno-inflamatório promove a progressiva destruiçáo dos tecidos de suporte e sustentaçáo dos dentes e, eventual, perda dentária (FLEMMIG, 1999; CHAPPLE; GENCO, 2013). Estima-se que aproximadamente de $10 \%$ da populaçáo do mundo sejam afetados pela forma mais severa da doença (FRENCKEN et al., 2017).

O diabetes mellitus tipo 2 (DM2) é considerado um fator de risco para o desenvolvimento da periodontite (CHAVARRY et al., 2009). DM2 é uma síndrome clínica com expressão fenotípica variável, sem etiologia específica, considerada uma doença de natureza poligênica caracterizada pela disfunção bi hormonal do pâncreas (SANTOS et al., 2014). A consequente desregulação dos mecanismos de homeostasia do nível sanguíneo de glicose, ao longo do tempo, promove dano, disfunção e falha de diferentes órgãos, especialmente olhos, rins, nervos, coração e vasos sanguíneos (INTERNATIONAL DIABETES FEDERATION, 2015).

Há uma associação positiva entre DM2 e o aumento do risco para o desenvolvimento de periodontite (CHAVARRY et al., 2009). Acredita-se que há no diabético uma resposta inflamatória mais persistente, relacionada a uma atividade osteoclástica prolongada, aumento de perda de inserção periodontal e consequente perda dentária (LIU et al., 2006; DEMMER et al., 2012; JIMENEZ et al., 2012). Por outro lado, a periodontite crônica aumenta em seis vezes o risco para a desregulação do controle glicêmico em indivíduos com diabetes (SEPPALA et al., 1993), além de estar associada a um maior risco para o desenvolvimento de complicaçóes do DM2 (STANKO; IZAKOVICOVA, 2012). Há evidências de que a terapia periodontal pode resultar em melhora do controle glicêmico (CHEE; PARK; BARTOLD, 2013) através da redução dos níveis sorológicos de citocinas inflamatórias (ARTESE et al., 2015) e de que a DP pode funcionar como um fator modificador para o diabetes (STANKO; IZAKOVICOVA, 2012), havendo, portanto, o estabelecimento de uma relação bidirecional entre ambos.

Considerando o caráter multidisciplinar inerente às patologias, principalmente no tocante ao DM2, uma abordagem que integre o cuidado desse grupo de pacientes poderia trazer benefícios mútuos (ELANGOVAN et al., 2014). No Brasil, a legislação 
estabelece diretrizes para a formação de linhas de cuidado dentro da Rede de Atenção à saúde das Pessoas com Doenças Crônicas, que objetiva prestar atenção integral a portadores de doenças crônicas (BRASIL, 2014). O gerenciamento do cuidado ao paciente com diabetes está inserido nesse contexto. $\mathrm{Na}$ prática, entretanto, há fragilidades para implementação de uma linha de cuidado voltada para a atenção integral a esse paciente (SANTOS et al., 2015). Uma delas diz respeito ao processo de trabalho dentro das equipes de saúde na Atenção Primária, havendo persistência do enfoque biomédico, ainda não superado (ZACHARIAS et al., 2016).

A fragmentação do modelo vigente torna-se evidente a leitura do Caderno de Atenção Básica no 36 (BRASIL, 2013). Nele há o reconhecimento do papel da DP como um fator de risco para a descompensação do diabetes, porém não há a sugestão de uma ação efetivamente multidisciplinar inerente ao seu fluxo de cuidado, na forma de diretrizes para implantaçáo de um protocolo para o atendimento integrado.

Por considerar o protocolo de cuidado como potencial ferramenta para o preenchimento dessa lacuna, o objetivo desta pesquisa é buscar, na literatura nacional e internacional, a existência de protocolos validados referentes a um fluxo de cuidado integral para o paciente periodontal com DM2, para que possam ser pontos de partida para recomendaçóes às açôes desenvolvidas no âmbito da atenção primária em saúde no país. ${ }^{1}$

\section{Metodologia}

Foi realizada revisão de literatura do tipo scoping (STANKO; IZAKOVICOVA, 2015), a partir das bases de dados eletrônicas Medline via Pubmed, Scientific Electronic Library Online (Scielo), Literatura Científica e Técnica da América Latina e Caribe (LILACS), Web of Science e Scopus. A pesquisa teve a finalidade de investigar a existência de protocolos validados que pudessem ser relacionados ao atendimento do paciente periodontal portador de DM2 na atenção primária em saúde. A questionamento que impulsionou essa pesquisa foi: há na literatura a descrição de protocolos validados para o atendimento integrado médico-odontológico do paciente diabético tipo 2 que possam ser adaptados para o contexto da atenção primária em saúde pública? 
Os critérios de elegibilidade para a seleção das publicaçôes, assim como termos de busca, selecionadas para essa revisão foram sumarizados no quadro 1. Foi pesquisada literatura nacional e internacional, publicada entre janeiro de 2012 e dezembro de 2019, com a finalidade de acessar evidências mais recentes a respeito do assunto, nas línguas inglesa, espanhola e portuguesa. Os descritores (DeCs, BVS) e meshes (Pubmed) foram, então, combinados através dos operadores boleanos AND, OR e AND NOT.

Quadro 1. Descritores utilizados no levantamento bibliográfico. Critérios de inclusão e critérios de exclusẫo utilizados para a seleçẫo dos artigos

\begin{tabular}{|c|c|c|}
\hline Termos relacionados à busca & $\begin{array}{c}\text { Descritores utilizados para } \\
\text { busca }\end{array}$ & Critérios de exclusão \\
\hline $\begin{array}{c}\text { Doença periodontal em } \\
\text { indivíduos diabéticos tipo 2. }\end{array}$ & $\begin{array}{c}\text { Diabetes mellitus tipo 2. } \\
\text { Atenção primária em saúde. }\end{array}$ & $\begin{array}{c}\text { Estudos restritos a doenças } \\
\text { gengivais. }\end{array}$ \\
$\begin{array}{c}\text { Controle metabólico dos } \\
\text { diabéticos. }\end{array}$ & $\begin{array}{c}\text { Saúde pública. } \\
\text { Doenças periodontais. Índice } \\
\text { glicêmico/glicemia. } \\
\text { Protocolo odontolón clínicos das áreas } \\
\text { atendimento para a diabéticos na } \\
\text { atençáo primária em saúde. }\end{array}$ & $\begin{array}{c}\text { Protocolos clínicos. Diretrizes } \\
\text { Estudos relacionados a fatores } \\
\text { genéticos e microbiológicos. }\end{array}$ \\
$\begin{array}{c}\text { Protocolos para atendimento clínica. } \\
\text { odontológico ao paciente } \\
\text { diabético. }\end{array}$ & Periodontite. Medicina. & Estudos em animais. \\
\hline
\end{tabular}

Fonte: elaboração própria.

Os artigos foram selecionados por dois avaliadores (LML, LMB), pelos títulos, havendo exclusão daqueles que não estavam relacionados a questão central da pesquisa. Foram selecionados, inicialmente, 236 artigos com descritores correspondentes ao assunto central da pesquisa (figura 1). 


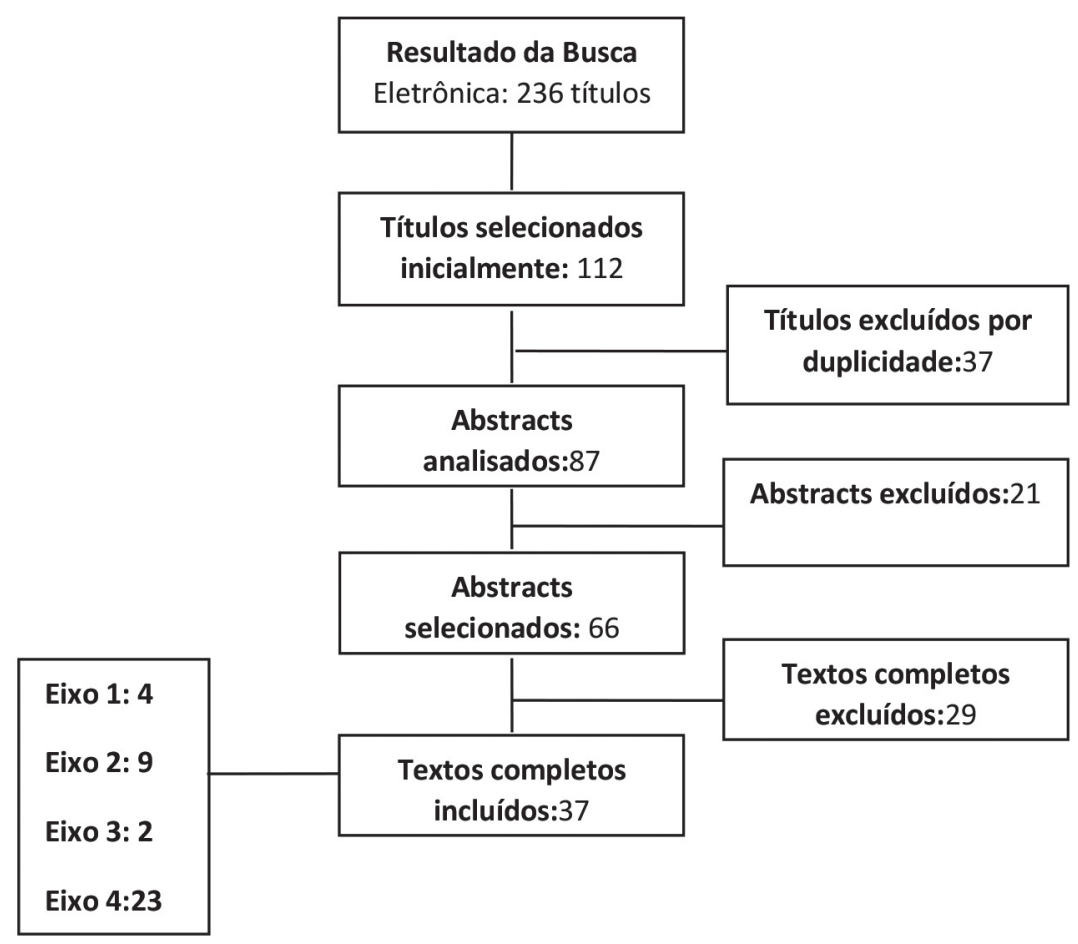

Fonte: elaboração própria.

Destes, 112 artigos foram eleitos, por relacionar diretamente os descritores DM2 e DP. Após a exclusão de 37 repetidos, restaram 87 para análise dos resumos. Nessa etapa, os artigos selecionados para a revisão deveriam estar em consonância com os seguintes eixos:

1) DM2 e DP na atenção primária em saúde;

2) Relação entre médico e dentista no cuidado integral do indivíduo com diabetes tipo 2, portador de DP, de maneira geral e no contexto da Atenção Primária em Saúde;

3) Existência de protocolos validados de atenção em saúde bucal ao indivíduo com diabetes tipo 2 e consensos publicados a respeito do assunto;

4) Evidências disponíveis para o tratamento odontológico do paciente com diabetes portador de DP. 
Após a leitura dos resumos, feita em duplicidade pelos mesmos avaliadores, houve a exclusão, após debate, daqueles que não poderiam ser colocados em nenhum dos eixos, por fugirem ao escopo da pesquisa; havendo discordância, um terceiro revisor foi consultado. Restaram 37 artigos para análise (quadro 2).

Quadro 2. Associaçóes médicas que adotaram recomendaçóes para o cuidado integrado médico-odontológico

\begin{tabular}{|c|c|}
\hline Associação Médica & Recomendação \\
\hline $\begin{array}{c}\text { Associação Americana do Diabetes (ADA): } \\
\text { Diretrizes (2016) }\end{array}$ & $\begin{array}{l}\text { Referência ao dentista para cuidados preventivos } \\
\text { e exame periodontal no início do tratamento; }\end{array}$ \\
\hline $\begin{array}{l}\text { Iniciativa Nacional de Educação do Diabetes } \\
\qquad(2016)\end{array}$ & Corrobora a ADA \\
\hline $\begin{array}{l}\text { Federação Internacional do Diabetes: Diretrizes } \\
\qquad(2009)\end{array}$ & $\begin{array}{l}\text { Recomendação quanto a conscientizaçáo do } \\
\text { diabético para higiene oral e risco de xerostomia }\end{array}$ \\
\hline $\begin{array}{c}\text { Consenso da Academia Americana de } \\
\text { Periodontologia (2013) }\end{array}$ & $\begin{array}{l}\text { Orientaçôes relativas ao cuidado periodontal do } \\
\text { paciente diabético }\end{array}$ \\
\hline Academia Americana de Medicina Oral (2015) & $\begin{array}{l}\text { Recomendaçóes para otimizar intervençôes e } \\
\text { reduzir o risco de cárie, doença periodontal e } \\
\text { infecçôes orais em pacientes com xerostomia, } \\
\text { diabetes, doença pulmonar obstrutiva crônica e } \\
\text { falha cardíaca congestiva. }\end{array}$ \\
\hline
\end{tabular}

Fonte: elaboração própria.

\section{Resultados e Discussão}

\section{DM2 e DP na Atenção Primária em Saúde}

A busca nos bancos de dados revelou limitada literatura com especificidade a respeito do assunto, tanto nacional quanto internacionalmente. Houve, nesse sentido, uma tendência a considerar o ambiente do consultório odontológico como potencial local para triagem de indivíduos com risco aumentado para o desenvolvimento do diabetes, indivíduos pré-diabéticos (HbA1c entre 5,7 e 6,4\%) e diabéticos (HbAlc $\geq$ 6,5\%) ainda não diagnosticados, dada a estreita correlação entre DP e DM2.

A prevalência de hiperglicemia não diagnosticada entre pacientes odontológicos foi investigada por Tantipoj et al. (2017). Um percentual superior a 30\% de indivíduos com alguma alteração do índice glicêmico foi encontrado em seu estudo. Foi estabelecida, ainda, correlação estatisticamente significativa entre o aumento do 
risco para hiperglicemia, perda dentária e aumento da profundidade de sondagem. Bould et al. (2017), em ensaio clínico desenvolvido no Reino Unido, estudaram a adesão, entre pacientes odontológicos, a duas ferramentas para triagem de indivíduos hiperglicêmicos: o FINDRISC, um questionário validado, considerado um tipo de triagem não invasivo, associado ao exame de hemoglobina glicada (HbA1c), considerada uma forma de triagem invasiva.

Os pacientes, selecionados pelo questionário como de maior risco para hiperglicemia, foram submetidos ao exame de HbAlc. Aqueles com resultados notadamente alterados foram encaminhados ao seu médico da atenção primária. Observou-se que 60\% dos participantes hiperglicêmicos, rastreados pela pesquisa, contataram o médico da atençáo primária para iniciar precocemente medidas de prevenção ou tratamento, sendo, portanto, demonstrada a aceitação dessa abordagem preventiva pelos mesmos. Ambas pesquisas asseveram a viabilidade do rastreio de pacientes hiperglicêmicos não diagnosticados no ambiente da consulta odontológica.

Uma análise do custo-efetividade foi realizada para esse tipo de intervençáo por Neidell, Lamster eShearer (2017). Esses autores demonstraram que o foco no tratamento de indivíduos com pré-diabetes tem, consideravelmente, melhor custo-efetividade do que o foco no tratamento de indivíduos com diabetes, e que a triagem de pacientes no ambiente do consultório odontológico é um meio econômico de identificação precoce de pacientes com diabetes e pré-diabetes. $\mathrm{O}$ estudo foi desenvolvido nos Estados Unidos, país pertencente à regiấo com maior prevalência de indivíduos com diabetes do mundo e onde se estima um gasto sanitário com DM que representa 51,7\% do gasto mundial com a doença de acordo com a Federação Internacional de Diabetes (INTERNATIONAL DIABETES FEDERATION, 2015).

Seguindo a mesma linha, Solowiej-Wedderburn, Ide e Pennington (2017) investigaram o custo-efetividade que a terapia periodontal não cirúrgica e sua manutenção poderiam representar para o provedor de saúde no Reino Unido. Seu trabalho demonstrou que há elevação de custo, porém os benefícios a saúde atribuídos a redução da HbA1c em pacientes com DM2 seriam suficientes para justificar o custo adicional com esse subgrupo de pacientes, antes mesmo de considerar os benefícios a saúde oral. Os autores reforçam o argumento da necessidade de inclusão da saúde periodontal nas diretrizes clínicas para o manejo do DM2, face ao acúmulo de evidências referentes aos benefícios trazidos pela terapia periodontal para esse grupo (HASUIKE et al., 2017). 
No Brasil, a integralidade do atendimento é uma diretriz a ser observada para a organização das açóes e serviços públicos de saúde (BRASIL, 1988; 1990) diferentemente de outros países. O envelhecimento populacional (MENDES et al., 2012) e a transição nutricional, entretanto, fazem com que a prevalência da DM2 aumente de forma exponencial, adquirindo característica epidêmicas no país (SARTORELLI et al., 2003).

Um estudo, que analisou a carga global da doença no Brasil, demonstrando que DM2 representa 5\% do total de doenças, colocada entre os cinco agravos mais importantes, sendo sua participação mais expressiva entre as doenças crônicas não transmissíveis (COSTA et al., 2017). Esse contexto aproxima a realidade desse problema específico com a de outros países que o vêm enfrentando há mais tempo.

\section{Relação entre médico e dentista no cuidado integral com diabetes tipo 2, portador de DP, de maneira geral e no contexto da Atenção Primária em Saúde}

Poucos autores investigaram o estabelecimento da relaçâo entre a integração dos cuidados médico e odontológico para o atendimento ao paciente diabético tipo 2 (LIN et al., 2014; GLURICH et al., 2017). Seus resultados, entretanto, foram convergentes ao documentar o uso potencial da equipe de saúde bucal em diferentes pontos de atenção para o monitoramento desses pacientes. Evidenciou-se, ainda, incipiente difusão dos conhecimentos acumulados ao longo de vinte anos de pesquisas da relação bidirecional entre diabetes e periodontite na área médica.

Outros estudos analisaram o nível de conhecimento a respeito da relação entre diabetes e periodontite, entre profissionais envolvidos com a prática de cuidados a esse grupo e entre os pacientes portadores de diabetes mellitus (COSTA et al., 2017; WEINSPACH et al., 2013; POUDEL et al., 2017). Ficou documentada a falha na transmissão de informaçóes referentes ao papel da saúde bucal para o controle da doença, tanto em meio aos profissionais médicos (COSTA et al., 2017), quanto aos pacientes, sobretudo diabéticos tipo 2 (GLURICH et al., 2017).

No Brasil, alguns autores identificaram dificuldades semelhantes, registrando uma ausência de acompanhamento regular da saúde bucal dos pacientes diabéticos por parte de cirurgiôes-dentistas atuantes no Saúde da Família (SARDENBERG et al., 2011; SOUZA et al., 2016). 
Como estratégia para a solução desses problemas, alguns autores destacam a necessidade de incorporar a saúde bucal ao escopo de diretrizes voltadas ao cuidado do paciente com diabetes nos guias de prática clínica, além da promoção de educação interprofissional, com a colaboração entre as áreas médica e odontológica (COSTA et al, 2016; GLURICH; NYCZ; ACHARYA, 2017; WEINSPACH et al., 2013). Poudel et al. (2017) foram um pouco além, salientando o papel potencial que pode ser desempenhado pelas enfermeiras da Estratégia Saúde da Família, treinadas para promover saúde bucal entre pacientes com diabetes.

A importância do estabelecimento da coordenação do cuidado para o gerenciamento clínico do paciente com diabetes é enfatizada por outros autores. Elangovan et al. (2014), consideram o consultório odontológico como um cenário ideal que oportuniza a captação de pacientes com diabetes não diagnosticados e também o monitoramento do controle glicêmico em portadores de diabetes, durante as consultas da terapia periodontal de suporte (TPS) do paciente, que tem frequência comumente estipulada entre três e seis meses após a conclusão do tratamento inicial. Ahdi et al. (2015), por outro lado, sugerem que a troca contínua de informaçôes entre o médico do paciente e seu dentista pode ser uma ferramenta útil, para a identificação de pacientes que tenham diabetes com pobre saúde bucal, sobretudo aqueles portadores de DP não diagnosticada e, consequentemente, não tratada.

Uma experiência de abordagem colaborativa foi relatada por Ota et al. (2013) para o tratamento de pacientes com diabetes recém-diagnosticados, portadores de periodontite. Em seu estudo, foram avaliados parâmetros periodontais, HbAlc e glicemia capilar, antes da terapia periodontal inicial (TPI), após e durante a TPS (entre 3-6 meses após a TPI,) ao mesmo tempo que os pacientes recebiam tratamento médico para o diabetes, que consistiu na prescrição de medicamentos e instrução quanto a dieta e exercícios.

Os autores observaram redução de $2,5 \%$ do índice glicêmico, muito superior à redução de 0,29\% relatada em metanálise que investigou o efeito isolado do tratamento periodontal sobre o índice glicêmico (2015). Importante mencionar que o estudo em questão apresentou limitaçôes, como o tamanho reduzido da amostra e ausência de grupo controle, porém esses resultados abrem espaço para o estabelecimento de questionamentos a serem respondidos.

No Brasil, estima-se que $5 \%$ dos portadores de diabetes mellitus pertençam à faixa etária entre 30-59 anos (BRASIL, 2013) e que, em torno, de 82,2\% apresente alguma 
condição não compatível com higidez periodontal dentro da mesma faixa (BRASIL, 2012). Talvez este último dado seja explicado pelo fato de que $47 \%$ dos brasileiros pertencentes à amostra da última Pesquisa Nacional de Saúde (BRASIL, 2015) não utilizavam escova, pasta ou fio dental para a limpeza dos dentes ou a uma provável ineficiência do serviço de saúde bucal (MACÊDO; CHAVES; FERNANDES, 2016). Essas informaçôes deixam transparecer a necessidade do desenvolvimento de estratégias para o enfrentamento das necessidades de saúde atuais e futuras da população brasileira, quando se considera a fase de transição demográfica vivida pelo país (VASCONCELOS; GOMES, 2012).

A bibliografia pesquisada denota uma tendência internacional a se levantar questionamentos a respeito da rotina de cuidados voltados ao atendimento do paciente com diabetes, diante das evidências acumuladas em torno dos benefícios adicionais proporcionados pela atenção em saúde bucal, quando integrada ao seu plano de tratamento. Para Dale et al. (2014), há uma consciência muito limitada da relação entre periodontite e efeitos adversos no diabetes entre pacientes portadores da doença e profissionais da saúde que parece um padrão internacional. Este fato, na opinião dos mesmos autores, pode refletir limitaçóes na comunicação e integração da interface entre odontologia e serviços médicos gerais e lacunas nas formaçôes das profissóes de saúde.

As equipes de saúde precisam estar cientes das principais manifestaçôes orais do diabetes, para que possam tornar-se mais eficientes na prevenção, diagnóstico inicial e, quando indicado, referenciamento para um periodontista. Halai (2014) menciona a necessidade de pesquisas para a descoberta de novos caminhos para a condução dessa interrelação profissional.

Um panorama a respeito do atual status da integração entre os cuidados médicoodontológico do paciente com diabetes, no âmbito da atenção primária, foi realizado por Glurich, Nycz e Acharya (2017). Os autores realizaram uma revisão dos principais documentos considerados como referência para a tomada de decisôes clínicas para o tratamento médico do paciente diabético, identificando aqueles que apresentavam alguma recomendação em relação à odontologia (quadro 3). No Brasil, as diretrizes da Sociedade Brasileira de Diabetes (SBD) não mencionam qualquer recomendação referente à saúde bucal (SOCIEDADE BRASILEIRA DE DIABETES, 2017).

Os mesmos autores (GLURICH et al., 2017) detectaram ainda potenciais barreiras à integração do cuidado ao paciente diabético entre as equipes de saúde médico- 
odontológica. É verdadeiro que muitos fatores ali colocados não seriam aplicáveis à realidade brasileira, considerando a singularidade do seu sistema de saúde e legislação.

\section{Quadro 3. Guias com recomendaçôes para práticas clínicas sugeridos pela Academia Americana de Periodontia e Federaçáo Europeia de Periodontia}

\begin{tabular}{|c|c|}
\hline $\begin{array}{c}\text { Recomendaçáo para médicos e demais } \\
\text { profissionais da saúde }\end{array}$ & Recomendação para dentistas \\
\hline $\begin{array}{l}\text { Pacientes com diabetes deveriam ser informados } \\
\text { de que a presença da doença periodontal } \\
\text { dificulta o controle glicêmico e aumenta o risco } \\
\text { para complicaçóes renais e cardíacas do diabetes }\end{array}$ & $\begin{array}{l}\text { Pacientes diabéticos deveriam ser informados de } \\
\text { que a doença aumenta o risco para periodontite. } \\
\text { Assim como de que se eles já tiverem periodontite, } \\
\text { há maior dificuldade para o controle glicêmico e } \\
\text { aumento do risco para complicaçôes do diabetes. }\end{array}$ \\
\hline $\begin{array}{l}\text { Como parte da sua avaliação inicial, pacientes } \\
\text { com diabetes tipo } 1 \text {, tipo } 2 \text { e diabetes gestacional } \\
\text { deveriam receber uma avaliaçáo completa da } \\
\text { saúde bucal. }\end{array}$ & $\begin{array}{l}\text { Pacientes diagnosticados com diabetes tipo } \\
1,2 \text { e gestacional, deveriam receber exame } \\
\text { bucal completo, além de detalhada avaliação } \\
\text { periodontal. }\end{array}$ \\
\hline $\begin{array}{l}\text { Recomendaçáo de encaminhamento do paciente } \\
\text { ao seu dentista para pelo menos } 1 \text { exame bucal } \\
\text { anual, mesmo em pacientes diabéticos que náo } \\
\text { tenham doença periodontal. }\end{array}$ & $\begin{array}{l}\text { Caso haja diagnóstico de periodontite, ela } \\
\text { deveria ser apropriadamente tratada. Caso não } \\
\text { haja diagnóstico de periodontite, inicialmente, } \\
\text { os pacientes devem ser colocados em regime } \\
\text { de cuidados preventivos e monitorados } \\
\text { preventivamente para mudanças periodontais. }\end{array}$ \\
\hline $\begin{array}{l}\text { Pacientes diabéticos que apresentem sinais } \\
\text { e sintomas como: dentes com mobilidade, } \\
\text { espaçados ou espalhados, abcessos ou supuração } \\
\text { gengival, requerem encaminhamento ao } \\
\text { periodontista para avaliação imediata. }\end{array}$ & $\begin{array}{l}\text { Pacientes com condição aguda/infecção } \\
\text { periodontal requer pronto tratamento. }\end{array}$ \\
\hline $\begin{array}{l}\text { Pacientes com extensas perdas dentárias } \\
\text { deveriam ser encorajados a buscar reabilitação } \\
\text { bucal, para reestabelecimento da mastigaçáo } \\
\text { adequada para apropriada nutrição. }\end{array}$ & $\begin{array}{l}\text { Pacientes com extensiva perda dentária, deveria } \\
\text { ser encorajado a buscar reabilitação oral, para } \\
\text { reestabelecimento da mastigação adequada para } \\
\text { apropriada nutrição. }\end{array}$ \\
\hline $\begin{array}{l}\text { Pacientes deveriam receber educação em saúde } \\
\text { bucal. }\end{array}$ & $\begin{array}{l}\text { Todos os pacientes com diabetes deveriam } \\
\text { receber educaçáo em saúde bucal. }\end{array}$ \\
\hline $\begin{array}{l}\text { Pacientes diabéticos deveriam ser avisados } \\
\text { quanto a outras manifestaçóes bucais do } \\
\text { diabetes, como xerostomia e ardência bucal, } \\
\text { além da possibilidade do desenvolvimento de } \\
\text { infecções fúngicas, além da cicatrização de } \\
\text { feridas retardada. }\end{array}$ & $\begin{array}{l}\text { Pacientes sem o diagnóstico de diabetes, mas como } \\
\text { óbvios fatores de risco para diabetes tipo } 2 \text { e sinais } \\
\text { de periodontite, devem ser informados sobre seu } \\
\text { risco para diabetes, avaliação do índice glicêmico } \\
\text { (HbA1c) e/ou ser referido ao seu médico, para } \\
\text { diagnóstico apropriado e tratamento. }\end{array}$ \\
\hline
\end{tabular}

Fonte: adaptado de Chapple e Genco (2013). 


\section{Existência de protocolos validados de atenção em saúde bucal ao indivíduo com diabetes tipo 2 e consensos publicados a respeito do assunto}

Náo foi encontrado, nos limites da literatura pesquisada, qualquer protocolo validado para o atendimento odontológico de pacientes com diabetes, nem para o contexto geral, nem para o da atenção primária em saúde. O que se obteve foi uma revisão de literatura com propostas de diretrizes para o acolhimento e tratamento desses pacientes (AL-NOWAISER et al., 2014). Foi encontrado, entretanto, um posicionamento por parte da Academia Americana de Periodontia e da Federação Europeia de Periodontia sobre a relaçáo entre diabetes e periodontite na forma de um consenso (CHAPPLE; GENCO, 2013).

De acordo com esse documento, há plausibilidade biológica de que a inflamação crônica não resolvida derivada da DP impacta no controle do diabetes (elevando a HbA1c) e suas complicações, bem como na função das células $\beta$, resistência à insulina e desenvolvimento do DM2. A hiperglicemia, por outro lado, afeta negativamente o desfecho da DP, contribuindo para o aumento da sua severidade (CHAPPLE; GENCO, 2013). Como consequência, o controle glicêmico em pacientes periodontais seria mais difícil. Um resumo do guia com sugestôes de práticas clínicas referentes à saúde bucal, para o médico e demais profissionais da saúde e para o dentista, está listado na figura 3.

\section{Evidências disponíveis sobre abordagens de tratamento odontológico ao paciente periodontal com DM2}

Muitos ensaios clínicos comparando diversas abordagens terapêuticas para o tratamento periodontal do paciente com diabetes estáo publicados (RAMAN et al., 2014; AL-NOWAISER et al., 2014; KAUR et al., 2015; SANTOS et al., 2016). Pensando na profusão de pesquisas realizadas, Botero, Rodríguez, Agudelo-Suarez (2016) e Hasuike et al. (2017) realizaram overviews sem e com meta-análise sobre ensaios clínicos que discutiram os efeitos do tratamento periodontal em indivíduos com diabetes. Esses autores concluíram que há um efeito do tratamento periodontal na melhora do HbAlc em pacientes com diabetes de tamanho modesto, porém significativo do ponto de vista estatístico.

Outros autores (KAUR et al., 2015; ALTAMASH; KLINGE; ENGSTROM, 2015) avaliaram, por meio de ensaios clínicos, o impacto da terapia periodontal sobre o índice glicêmico entre indivíduos com e sem diabetes com periodontite. 
Demonstraram em seus estudos que o tratamento periodontal está associado a uma significativa melhora na glicemia de indivíduos com periodontite de moderada a severa, independentemente do tipo de hipoglicemiante oral utilizado, e que o acompanhamento periodontal deve exceder os três meses para um significativo efeito redutor do índice glicêmico.

Ensaio clínico realizado por Artese et al. (2015) comparou o efeito do controle do biofilme supragengival, apenas, com a realização de terapia periodontal convencional, com raspagem e alisamento radicular, em marcadores sorológicos inflamatórios de indivíduos com DM2, portadores de periodontite crônica. O estudo demonstrou que houve ganho clínico de inserção em ambos os grupos, mas naquele que recebeu terapia periodontal convencional, houve mais benefícios para melhora dos parâmetros periodontais.

Em suma, eles opinam que a terapia periodontal convencional deve ser priorizada para o controle da carga inflamatória em indivíduos com DM2. No estudo de Raman et al. (2014), o efeito da instrução de higiene oral somente, nos níveis glicêmicos de pacientes com DM2, foi semelhante - ou seja, pacientes submetidos a terapia periodontal tiveram maior redução da Hb1Ac. Os autores argumentaram que ensinar técnicas de controle de placa ao paciente com DM2 e corrigir escovação inadequada são capazes de, clinicamente, traduzir-se em melhora do controle metabólico, uma vez que em seu estudo observaram redução de $0,5 \%$ na hemoglobina glicada dos pacientes que receberam apenas instrução de higiene oral (RAMAN et al., 2014). No entanto, López et al. (2014) demonstraram que a resposta ao controle de placa e a raspagem supragengival é similar entre pacientes periodontais com ou sem diabetes, havendo prevenção da progressão da DP em pacientes com bom e com pobre controle glicêmico.

Há, na literatura, muitas revisões sobre a relação entre o tratamento periodontal e o controle glicêmico sustentando o papel do tratamento periodontal na redução do índice glicêmico de pacientes com diabetes. $\mathrm{O}$ tratamento da doença periodontal seria comparável à adição de uma segunda medicação hipoglicemiante, havendo, portanto, potencial para reduzir custos inerentes à terapêutica desses pacientes (LIEW et al., 2013; SGOLASTRA, 2013; SIMPSON et al., 2015; HASUIKE et al., 2017; D’AIUTO et al., 2018). O tratamento periodontal seria, portanto, um adjuvante a um planejamento terapêutico maior, multidisciplinar e mais efetivo, tanto do ponto de vista médico, quanto odontológico, por possibilitar melhor racionalização do tempo de atendimento de cada profissional dedicado a esse paciente. 
Revisóes sistemáticas sugerem que a antibioticoterapia, usada como um adjunto à terapia periodontal não cirúrgica, traz benefícios adicionais na redução da profundidade de sondagem $(0,22 \mathrm{~mm})$, sangramento a sondagem $(4 \%)$ e ganho clínico de inserção (em sítios com profundidade de sondagem superiores a $5 \mathrm{~mm}$ na baseline) quando comparada a raspagem e alisamento radicular somente (D'AIUTO et al., 2018; SANTOS et al., 2015). Esses resultados devem ser tomados com cautela, considerando o risco para o desenvolvimento de resistência microbiana.

Com relação aos antimicrobianos selecionados, há ensaios clínicos (GILOWSKI et al., 2012; BOTERO et al., 2013; AL-NOWAISER et al., 2014; MIRANDA et al., 2014; SANTOS et al., 2015; ROVAI et al., 2016; TAMASHIRO et al., 2016) que investigaram o efeito da associação entre metronidazol e amoxicilina, ou doxiciclina, ou azitromicina, dentre outros, e terapia periodontal básica no longo prazo. Notouse redução do risco para futura progressão da doença, diminuição da resposta inflamatória, além do aumento da previsibilidade para os resultados do tratamento.

Essas evidências não demonstraram, entretanto, que há contribuição da antibioticoterapia, como adjuvante, para a redução nos níveis glicêmicos de indivíduos portadores de DM2. Está estabelecido que a existência da DP interfere no controle glicêmico de pacientes portadores de diabetes. Há evidências de que o controle da DP pode funcionar como uma ferramenta adicional, dentro de programas maiores para o planejamento terapêutico desse grupo de pacientes.

Um estudo (SAENGTIPBOVORN; TANEEPANICHSKUL, 2015) sobre o efeito de um programa de educação em saúde voltado para diabéticos utilizou técnicas da teoria cognitivo comportamental voltadas para adoção de um estilo de vida mais saudável, com práticas de exercícios físicos e mudança na dieta, associadas à educação voltada para a higiene bucal e avaliação de parâmetros clínicos periodontais. Mesmo que esse ensaio não tenha avaliado o impacto específico do programa de saúde bucal sob o controle glicêmico dos pacientes investigados, houve melhora estatisticamente significante da glicemia e estado de saúde periodontal dos pacientes investigados quando comparados aos controles que não participaram do programa.

Não houve, nos limites da literatura consultada, protocolos clínicos validados referentes ao cuidado integrado médico-odontológico do paciente com DM2. Tendo em vista os potenciais benefícios que poderiam ser alcançados no que se refere ao controle glicêmico desse grupo, recomenda-se o desenvolvimento de estudos que investiguem o impacto da integração dos cuidado médico-odontológico no controle 
glicêmico do paciente com DM2. Da mesma forma, devem ser realizados estudos que validem protocolos clínicos para a atenção multidisciplinar desse paciente no âmbito da atenção primária em saúde, além de avaliar seu custo-efetividade. Recomenda-se, ainda, maior disseminação de informaçôes referentes ao papel da saúde periodontal para o controle do DM2 entre profissionais médicos. ${ }^{2}$

\section{Referências}

AHDI, M. et al. Oral health information from the dentist to the diabetologist. Eur J Intern Med, v. 26, n. 7, p. 498-503, 2015. doi: 10.1016/j.ejim.2015.06.006

AL-NOWAISER, A. M. et al. Evaluation of adjunctive systemic doxycycline with non-surgical periodontal therapy within type 2 diabetic patients. Saudi Med J, v. 35, n. 10, p. 1203-9, 2014.

ALTAMASH, M.; KLINGE, B.; ENGSTROM, P. E. Periodontal treatment and HbA1c levels in subjects with diabetes mellitus. J Oral Rehabil, v. 43, n. 1, p. 31-8, 2016. doi: 10.1111/joor.12339.

ARTESE, H. P. et al. Periodontal therapy and systemic inflammation in type 2 diabetes mellitus: a meta-analysis. PLoS One, v. 10, n. 5, p. e0128344, 2015. doi: 10.1371/journal.pone.0128344.

ARTESE, H. P. C. et al. Supragingival biofilm control and systemic inflammation in patients with type 2 diabetes mellitus. Braz. oral res, v. 29, n. 1, p. 1-7, 2015. doi: 10.1590/18073107BOR-2015.vol29.0071.

BOtero, J. E.; RODRIGUEZ, C.; AGUDELO-SUAREZ, A. A. Periodontal treatment and glycaemic control in patients with diabetes and periodontitis: an umbrella review. Aust Dent J, v. 61, n. 2, p. 134-48, 2016. doi: 10.1111/adj.12413.

BOTERO, J. E. et al. Effects of periodontal non-surgical therapy plus azithromycin on glycemic control in patients with diabetes: a randomized clinical trial. J Periodontal Res, v. 48, n. 6, p. 706-12, 2013. doi: 10.1111/jre.12058.

BOULD, K. et al. Uptake of screening for type 2 diabetes risk in general dental practice: an exploratory study. Br Dent J, v. 222, p. 293-6, 2017. doi: 10.1038/sj.bdj.2017.174.

BRASIL. Ministério da Saúde. Secretaria de Atenção à Saúde. Secretaria de Vigilância em Saúde. SB Brasil 2010. Pesquisa Nacional de Saúde Bucal: resultados principais. Brasília: Ministério da Saúde, 2012. 116p. Disponível em: <https://bvsms.saude.gov.br/bvs/publicacoes/pesquisa_ nacional_saude_bucal.pdf.> Acesso em: 26 maio 2020.

. Constituição Federal da República Federativa do Brasil. Seção II-Da Saúde (arts.196 a 200). Brasília, DF: Presidência da República, 2016. Disponível em: <http://alerjln1.alerj.rj.gov. br/constfed.nsf/16adba33b2e5149e032568f60071600f/171f51d63db822a703256562006e66bc ?OpenDocument>. Acesso em: 26 maio 2020. 
. Lei n. 8.080, de 19 de setembro de 1990. Dispóe sobre as condições para a promoção, proteção e recuperação da saúde, a organização e o funcionamento dos serviços correspondentes e dá outras providências. Brasília, DF: Presidência da República, 2017. Disponível em: <http:// www.planalto.gov.br/ccivil_03/leis/L8080.htm>. Acesso em: 26 maio 2020.

. Ministério da Saúde. Gabinete do Ministro. Portaria no 483, de $1^{\circ}$ de abril de 2014. Redefine a Rede de Atenção à Saúde das Pessoas com Doenças Crônicas no âmbito do Sistema Único de Saúde (SUS) e estabelece diretrizes para a organização das suas linhas de cuidado. Sistema de Legislação da Saúde. 2014. Disponível em: <http://bvsms.saude.gov.br/bvs/ saudelegis/gm/2014/prt0483_01_04_2014.html>. Acesso em: 26 de maio 2020.

. Ministério da Saúde. Secretaria de Atenção à Saúde. Departamento de Atenção Básica. Estratégias para o cuidado da pessoa com doença crônica: diabetes mellitus. Ministério da Saúde, Secretaria de Atenção à Saúde, Departamento de Atenção Básica. Brasília. Ministério da Saúde, 2013 (Cad. Atenção Básica, n 36). Disponível em: <http://bvsms.saude.gov.br/bvs/publicacoes/ estrategias_cuidado_pessoa_diabetes_mellitus_cab36.pdf>. Acesso em: 20 maio 2020.

- Ministério do Planejamento O e G. Pequisa Nacional de Saúde. Instituto Brasileiro de Geografia e Estatística-IBGE, 2015, 105 p. Disponível em: <https://biblioteca.ibge.gov.br/ visualizacao/livros/liv94074.pdf>. Acesso em: 26 maio 2020.

. Ministério do Planejamento O e G. Pesquisa Nacional de Saúde. Instituto Brasileiro de Geografia e Estatística, 2014, 180 p. Disponível em: <ftp://ftp.ibge.gov.br/PNS/2013/pns2013. pdf>. Acesso em: 26 maio 2020.

CHAPPLE, I. L.; GENCO, R. Diabetes and periodontal diseases: consensus report of the Joint EFP/AAP Workshop on Periodontitis and Systemic Diseases. J Periodontol, v. 84, n. 4, p. S10612, 2013. doi: 10.1902/jop.2013.1340011.

CHAVARRY, N. G. et al. The relationship between diabetes mellitus and destructive periodontal disease: a meta-analysis. Oral Health Prev Dent, v. 7, n. 2, p. 107-27, 2009.

CHEE, B.; PARK, B.; BARTOLD, P. M. Periodontitis and type II diabetes: a two-way relationship. Int J Evid Based Healthc, v. 11, n. 4, p. 317-29, 2013. doi: 10.1111/1744-1609.12038.

COSTA, A. F. et al. Carga do diabetes mellitus tipo 2 no Brasil. Cad. Saúde Pública, Rio de Janeiro, v. 33, n. 2, p. e00197915, 2017. doi: 10.1590/0102-311x00197915.

COSTA, R. M. et al. The diabetic patient in the dental clinic: care and treatment guidelines. Rev Bras Cien Saude, v. 20, n. 4, p. 333-40, 2016. doi: 10.4034/RBCS.2016.20.04.11.

DALE, J. et al. Oral health: A neglected area of routine diabetes care? Br J Gen Pract, v. 64, p. 103-4, 2014. doi: 10.3399/bjgp14X677301.

DEMMER, R. T. et al. The influence of type 1 and type 2 diabetes on periodontal disease progression: prospective results from the Study of Health in Pomerania (SHIP). Diabetes Care, v. 35, n. 10, p. 2036-42, 2012. doi: $10.2337 /$ dc11-2453. 
ELANGOVAN, S. et al. A Framework for Physician-Dentist Collaboration in Diabetes and Periodontitis. Clinical Diabetes, v. 32, p. 188-92, 2014. doi: 10.2337/diaclin.32.4.188.

FLEMMig, T. F. Periodontitis. Ann Periodontol, v. 4, n. 1, p. 32-8, 1999.

FRENCKEN, J. E. et al. Global epidemiology of dental caries and severe periodontitis: a comprehensive review. J Clin Periodontol, v. 44, n. 18, p. S94-s105, 2017. doi: 10.1111/jcpe.12677. GILOWSKI, L. et al. Efficacy of short-term adjunctive subantimicrobial dose doxycycline in diabetic patients-randomized study. Oral Dis, v. 18, n. 8, p. 763-70, 2012. doi: 10.1111/j.16010825.2012.01943.x.

GLURICH, I.; NYCZ, G.; ACHARYA, A. Status Update on Translation of Integrated Primary Dental-Medical Care Delivery for Management of Diabetic Patients. Clin Med Res, v. 15, n. 1-2, p. 21-32, 2017. doi: 10.3121/cmr.2017.1348.

HALAI, H. Diabetes: multi-disciplinary approaches. Braz. Dente. J, v. 216, n. 2, p. 48. doi: 10.1038/sj.bdj.2014.7.

HASUIKE, A. et al. Systematic review and assessment of systematic reviews examining the effect of periodontal treatment on glycemic control in patients with diabetes. Med Oral Patol Oral Cir Bucal, v. 22, n. 2, p. e167-e76, 2017. doi: 10.4317/medoral.21555.

INTERNATIONAL DIABETES FEDERATION. IDF Diabetes Atlas. Brussels: International Diabetes Federation, 2015. 140p. Disponível em: https:/www.idf.org/e-library/epidemiologyresearch/diabetes-atlas/13-diabetes-atlas-seventh-edition.html. Acesso em: 30 maio d 2020.

JIMENEZ, M. et al. Type 2 diabetes mellitus and 20 year incidence of periodontitis and tooth loss. Diabetes Res Clin Pract, v. 92, n. 3, p. 494-500, 2012. doi: 10.1016/j.diabres.2012.09.039.

KAUR, P. K. et al. Periodontal and glycemic effects of nonsurgical periodontal therapy in patients with type 2 diabetes stratified by baseline HbA1c. J Oral Sci, v. 54, n. 3, p. 201-11, 2015. doi: 10.2334/josnusd.57.201.

LIEW, A. K. et al. Effect of non-surgical periodontal treatment on HbA1c: a meta-analysis of randomized controlled trials. Aust Dent J, v. 58, n. 3, p. 350-7, 2013. doi: 10.1111/adj.12091.

LIN, H. et al. Knowledge, awareness, and behaviors of endocrinologists and dentists for the relationship between diabetes and periodontitis. Diabetes Res Clin Pract, v. 106, n. 3, p. 428-34, 2014. doi: 10.1016/j.diabres.2014.09.008.

LIU, R.etal.Diabetesenhancesperiodontalbonelossthroughenhanced resorptionanddiminished bone formation. J Dent Res, v. 85, n. 6, p. 510-4, 2006. doi: 10.1177/154405910608500606.

LÓPEZ, N. J. et al. Routine prophylaxes every 3 months improves chronic periodontitis status in type 2 diabetes. J Periodontol, v. 85, n. 7, p. e232-40, 2014. doi: 10.1902/jop.2013.130400. 
MACÊDO, M. S. R.; CHAVES, S. C. L.; FERNANDES, A. L. D. C. Investments and costs of oral health care for Family Health Care. Rev Saude Publica, São Paulo, v. 50, p. 41, 2016. doi: 10.1590/s1518-8787.2016050005771.

MENDES, A. D. C. G. et al. Assistência pública de saúde no contexto da transição demográfica brasileira: exigências atuais e futuras. Cad de Saude Publica, v. 28, p. 955-64, 2012. doi: 10.1590/ S0102-311X2012000500014.

MIRANDA, T. S. et al. Metronidazole and amoxicillin as adjuncts to scaling and root planing for the treatment of type 2 diabetic subjects with periodontitis: 1-year outcomes of a randomized placebo-controlled clinical trial. J Clin Periodontol, v. 41, n. 9, p. 890-9, 2014. doi: $10.1111 /$ jre.12058.

NEIDELL, M.; LAMSTER, I.; SHEARER, B. Cost-effectiveness of diabetes screening initiated through a dental visit. Community Dent Oral Epidemiol, v. 45, n. 3, p. 274-80, 2017. doi: $10.1111 /$ cdoe.12286.

OTA, M. et al. A Collaborative Approach to Care for Patients with Periodontitis and Diabetes. Bull Tokyo Dent Coll, v. 54, p. 51-7, 2013. doi: 10.2209/tdcpublication.54.51.

POUDEL, P. et al. Knowledge and practices of diabetes care providers in oral health care and their potential role in oral health promotion: A scoping review. Diabetes Res Clin Pract, v. 130, p. 266-77, 2017. doi: 10.1016/j.diabres.2017.06.004.

RAMAN, R. P. et al. Effect of nonsurgical periodontal therapy verses oral hygiene instructions on type 2 diabetes subjects with chronic periodontitis: a randomised clinical trial. BMC Oral Health, v. 14, n. 1, p.79, 2014. doi: 10.1186/1472-6831-14-79.

ROVAI, E. S. et al. Efficacy of Local Antimicrobials in the Non-Surgical Treatment of Patients With Periodontitis and Diabetes: A Systematic Review. J Periodontol, v. 87, n. 12, p. 1406-17, 2016. doi: 10.1902/jop.2016.160214.

SAENGTIPBOVORN, S.; TANEEPANICHSKUL, S. Effectiveness of lifestyle change plus dental care program in improving glycemic and periodontal status in aging patients with diabetes: a cluster, randomized, controlled trial. J Periodontol, v. 86, n. 4, p. 507-15, 2015. doi: 10.1902/jop.2015.

SANTOS, C. M. et al. Systemic Antibiotics in Periodontal Treatment of Diabetic Patients: A Systematic Review. PLoS One, v. 10, n. 12, p. e0145262, 2015. doi: 10.1371/journal.pone.0145262.

SANTOS, R. A.; LYRA, R.; CAVALCANTI, N. Diabetes mellitus e doenças cardiovasculares. São Paulo: AC Farmacêutica, 2014.

SANTOS, R. S. A. F. et al. Rede de Atenção à Saúde ao portador de Diabetes Mellitus: uma análise da implantação no SUS em Recife (PE). Saúde debate, Rio de Janeiro, v. 39, n. esp, p. 268-282, dez. 2015. doi: 10.5935/0103-1104.2015S005368. 
SARDENBERG, C. H. et al. Knowledge and coduct of the endocrinologists in the relationship between diabetes mellitus and periodontal disease. Periodontia, v. 21, n. 4, p. 60-65.

SARTORELLI, D. S.; FRANCO, L. J. Tendências do diabetes mellitus no Brasil: o papel da transição nutricional. Cad de Saude Publica, Rio de Janeiro, v. 19, supl. 1, p. S29-S36, 2003. doi: 10.1590/S0102-311X2003000700004.

SEPPALA, B.; SEPPALA, M.; AINAMO, J. A longitudinal study on insulin-dependent diabetes mellitus and periodontal disease. J Clin Periodontol, v. 20, n. 3, p. 161-5, 1993. doi: 10.1111/j.1600-051x.1993.tb00338.x.

SGOLASTRA, F. et al. Effectiveness of periodontal treatment to improve metabolic control in patients with chronic periodontitis and type 2 diabetes: a meta-analysis of randomized clinical trials. J Periodontol, v. 84, n. 7, p. 958-73, 2013. doi: 10.1902/jop.2012.120377.

SIMPSON, T. C. et al. Treatment of periodontal disease for glycaemic control in people with diabetes mellitus. Cochrane Database Syst Ver, v. 11, p. Cd004714, 2015. doi: $10.1002 / 14651858$.

SOCIEDADE BRASILEIRA DE DIABETES. Conduta Terapêutica no Diabetes Tipo 2: Algoritmo SBD 2017. São Paulo: Sociedade Brasileira de Diabetes, 2017. 40 p.

SOLOWIEJ-WEDDERBURN, J.; IDE, M.; PENNINGTON, M. Cost-effectiveness of nonsurgical periodontal therapy for patients with type 2 diabetes in the UK. J Clin Periodontol, v. 44, n. 7, p. 700-7, 2017. doi: 10.1111/jcpe.12746.

SOUZA, C. et al. Visão do paciente sobre a relação bilateral entre o diabetes mellitus e as doenças periodontais. Rev Bras Odontol, v. 73, n. 4, p. 288-92, out./dez. 2016. doi: 10.18363/ rbo.v73n4.p.288.

STANKO, P.; IZAKOVICOVA, H. L. Bidirectional association between diabetes mellitus and inflammatory periodontal disease. A review. Biomedical Pap Med Fac Univ Palacky Olomouc Czech Republic, v. 158, n. 1, p. 35-8, 2014. doi: 10.5507/bp.2014.005.

TAMASHIRO, N. S. et al. Amoxicillin Plus Metronidazole Therapy for Patients with Periodontitis and Type 2 Diabetes: A 2-year Randomized Controlled Trial. J Dent Res, v. 95, n. 7, p. 829-36, 2016. doi: 10.1111/jcpe.12282.

TANTIPOJ, C. et al. Screening for Type 2 Diabetes Mellitus and Prediabetes Using Point-ofcare Testing for HBA1c Among Thai Dental Patients. Southeast Asian J Trop Med Public Health, v. 48, n. 2, p. 455-65, 2017.

VASCONCELOS, A. M. N.; GOMES, M. M. F. Transição demográfica: a experiência brasileira. Epidemiol. Serv. Saúde, Brasília, v. 21, n. 4, p. 539-548, dez. 2012. doi: 10.5123/ S1679-49742012000400003. 
WEINSPACH, K. et al. Level of information about the relationship between diabetes mellitus and periodontitis--results from a nationwide diabetes information program. Eur J Med Res, v. 18, n. 1, p. 6, 2013. doi: 10.1186/2047-783X-18-6.

ZACHARIAS, F. et al. Avaliação de estrutura e processo na atenção em diabetes mellitus. Medicina (Ribeirão Preto), v. 49, n. 2, p. 134-142, 2 abr. 2016. doi: 10.11606/issn.2176-7262. v49i2p134-142.

\section{Notas}

${ }^{1}$ Artigo resultante da monografia de conclusão de curso da autora Lívia Maria Lopes de Oliveira, intitulada "Tratamento Integrado de Pacientes Periodontais com Diabetes Mellitus tipo 2 na Atenção Primária em Saúde Pública", defendida no Centro de Ensino Superior Maceió (CESMAC) em 2017.

${ }^{2}$ V. V. Santana-Lima e A. Penteado contribuíram com a concepção e delineamento do estudo, revisão crítica relevante do conteúdo intelectual e aprovação da versão final. L. M. Oliveira realizou a análise e interpretação dos dados e redação do estudo. M. Barbosa e J. H. Negreiros contribuíram com a redação da versão final do artigo. Os autores declaram-se responsáveis por todos os aspectos do trabalho, no sentido de garantir que as questóes relacionadas à exatidão ou à integridade de qualquer parte do manuscrito sejam devidamente investigadas e resolvidas. 


\section{Abstract}

Integrated care of type 2 diabetic periodontal patient in Primary Care: scoping review

Objective: To investigate the existence of validated protocols for the care of periodontal patients with type 2 diabetes mellitus (DM2), to transform them into recommendations for actions that could be developed within the scope of primary health care. Methods: A scoping review was carried out, and the literature published from 2012 to 2019 was reviewed in the PubMed, Scielo, LILACS, Web of Science and Scopus databases, in English, Spanish and Portuguese. Results: 236 titles were found, of which 37 were selected for full reading. The articles were grouped into four axes, according to the similarity of approaches to the subject addressed. Conclusion: Studies investigating the impact of the integration of medical and dental care in the glycemic control of patients with DM2 and validating the clinical protocols for multidisciplinary care for this patient in the context of primary health care should be carried out.

Keywords: comprehensive health care; diabetes mellitus, type 2; periodontal diseases; clinical protocols; Primary Health Care. 